\title{
Reflexiones sobre reversibilidad: La reversibilidad entre la teoría y la praxis
}

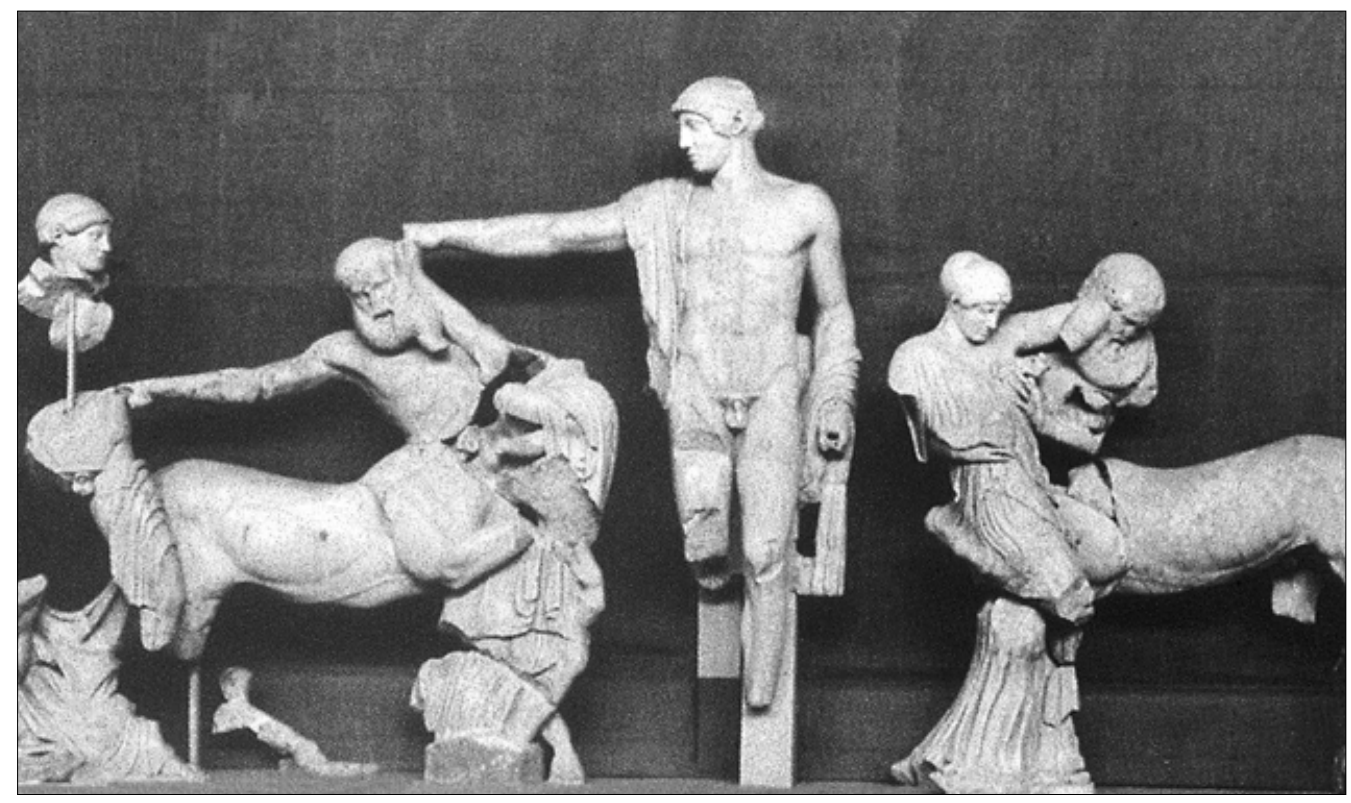

I. Templo de Zeus en Olimpia: frontón oeste: Combate de Centauros y lapitas. 470 - 460 a. J.C. Mármol. Olimpia, Museo Arqueológico.

\section{$\mathrm{M}^{\mathrm{a}}$ Isabel Fernández Medina}

Centro de Intervención del IAPH

En 1994 el Departamento de Conservación del Museo Británico organizó un ciclo de conferencias titulado Restauración, ¿es esto aceptable? La iniciativa tuvo tan buena acogida que en septiembre de 1999 se abordó otro argumento igualmente controvertido, como es la reversibilidad.

Reversibilidad y restauración son términos susceptible de diversas interpretaciones, y por tanto, capaces de estimular a los profesionales a participar en este debate.
Los conferenciantes provenían de muy variados sectores de la conservación, desde especialistas en conservación de pintura, de objetos de cristal, de papel, de arte contemporáneo, de textiles o de coches antiguos, contándose además con la participación de científicos dedicados a la investigación en la conservación de obras de arte; explorándose así el concepto de reversibilidad desde muy distintas perspectivas. Todos ellos contribuyeron con nuevas aportaciones a la revisión de las bases teóricas de este principio fundamental en el mundo de la conservación de bienes de interés cultural.

Generalmente se entiende que la reversibilidad es más una aspiración que una meta alcanzable, pero pocas personas realmente comprenden por qué esto es así. En el desarrollo del curso se demostró la existencia de insalvables obstáculos teóricos y prácticos con los que se enfrentan cada día los profesionales de la conservación de obras de arte. 
La gran afluencia de participantes, y la elevada cualificación de los ponentes provenientes de diversas instituciones, museos, y centros de investigación de reconocido prestigio, demostraron la gran aceptación e interés suscitado por este nuevo ciclo de conferencias; especialmente por tratarse de un tema del que se viene hablando desde hace tiempo y que requería una puesta al día, tanto desde un punto de vista teórico como práctico. Se creó un debate fomentado por las ideas aportadas y por las nuevas propuestas en torno a la posible aplicación de este principio, considerado hoy día como un clásico que, sin embargo, requiere una revisión en su utilidad y alcance real.

La puesta al día de uno de los criterios más utilizados en conservación que aparece en todos los tratados sobre teoría de la conservación de obras de arte, proyectos de conservación y restauración o informes técnicos de tratamientos ya realizados sobre obras determinadas, se hace necesario cuando somos conscientes de que teoría y práctica no van por el mismo camino. Es por tanto el momento de afrontar su análisis desde todas las perspectivas posibles y fundamentalmente desde el punto de vista teórico, de su aplicación práctica y de su adecuación terminológica; pues podemos plantearnos si estamos llamando a cada cosa por su nombre. La celebración de este curso ha supuesto una oportunidad de ponentes y participantes para exponer conclusiones basadas en experiencias profesionales, llegándose a proponer la idoneidad o no de la utilización del concepto de reversibilidad como principio teórico en el sector de la conservación de bienes culturales.

Tras la intervención de los ponentes pueden extraerse diversos posicionamientos teóricos que implican diferentes modos de afrontar un problema conceptual y lexicológico.

Appelbaum en su artículo "Criteria for treatement: reversibility", indica que la palabra "reversibilidad" debería ser usada para describir un proceso más que un material; un material no puede ser reversible.

Tomando como punto de partida esta definición considero que pueden establecerse los siguientes posicionamientos.

\section{Histórico}

El concepto de reversibilidad en conservación fue empleado por primera vez en el siglo XIX para la restauración de pinturas y ha continuado utilizándose hasta finales de la década de los 60 (Horie 1982). Quizás el primer autor que abordó el tema refiriéndose a la conservación de objetos fue Plederleith en 1934, quien mencionó la interferencia entre materiales y tratamientos y la dificultad de eliminar los adhesivos (Plenderleith 1934). Sobre el desarrollo del concepto de reversibilidad aplicado a la conservación de objetos, el término fue adoptado en los años 60 principalmente para la consolidación, reconstrucción y restauración (Rosenquist 1963; Unesco 1968; Oddy 1995). En los años 80 los conservadores aplicaban es- te concepto a todos los tratamientos de conservación, incluidos limpieza y estabilización (Oddy 1995).

\section{Filosófico}

Hace una década la reversibilidad significaba que cualquier material aplicado a un objeto debía ser fácilmente eliminable. Actualmente se incluyen todos los tratamientos aplicados a obras de arte. En consecuencia, los conservadores deben tener en cuenta, no sólo cambios físicos, sino también posibles cambios en su composición química y micro-estructura. El resultado es que muy pocos procesos son realmente reversibles, por tanto, determinar cómo actuar ante un caso concreto resulta no sólo una decisión técnica sino también ética.

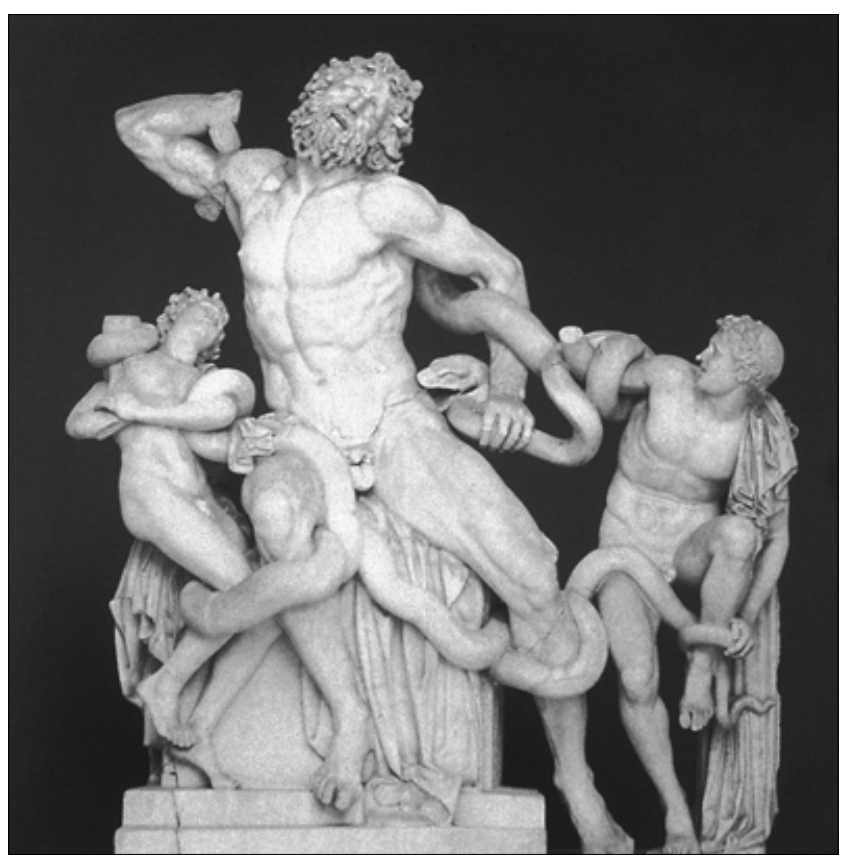

2. Laoconte y sus hijos. Adaptado (i) por los

Los conservadores-restauradores intentan encontrar métodos generales de tratamiento que puedan controlar con reglas como la "reversibilidad". Sin embargo, la existencia real de estas reglas se puede poner en duda ya que el valor dado a una obra de arte en un momento determinado del pasado depende de ideas contemporáneas, ideales y conceptos. Estos factores, por tanto, cambian con el curso de la historia: algunos ejemplos aparecen en los distintos periodos artísticos: Renacimiento, Neoclasicismo, Historicismo y Posmodernismo. Las nuevas ideas forman las bases para la evaluación de la cultura de tiempos pasados y ello conduce a la aparición de nuevos métodos de restauración. Al ser el tiempo irreversible, el término irreversibible no es aplicable a ninguna idea o materia y, en consecuencia, la reversibilidad resulta una utopía. Sin embargo, el intento de aproximarse a este ideal es absolutamente necesario.

Las crecientes dudas sobre la existencia de la reversibilidad, así como la común insistencia en su utopía, están escultores rodios Hagesandro, Atanodoro y Polidoro en el S. a partir de un grupo de bronce del S. III o de principios de

S. II a.l.C. Mármol. Roma Museos Vaticanos 
$\longleftarrow$
$\square$
$\square$ ligados a la pérdida de los valores tradicionales en la cultura moderna, y ello es debido al hecho de que nosotros, contemporáneos, no estamos seguros sobre la continuidad de los modos culturales de pensamiento.

Por otra parte, la creencia en la reversibilidad demuestra la inseguridad sobre la importancia y corrección de nuestras acciones y, además, responde a la ilusión de que algo pueda ser "deshecho", lo que puede llevar a una pérdida de responsabilidad. Así, como afirma Andrew Oddy, la utilización del concepto de reversibilidad sirve de justificación de los procesos de limpieza, muchas veces llevados a cabo por el deseo de los restauradores de demostrar sus habilidades en la conservación de obras de arte.

En este sentido, Nigel J.Seeley (p.161) intenta demostrar que el valor evidente de las obras de arte se extiende a todas sus propiedades, tanto tangibles como intangibles, las cuales están sujetas a cambios producidos por los procesos naturales y por las in-

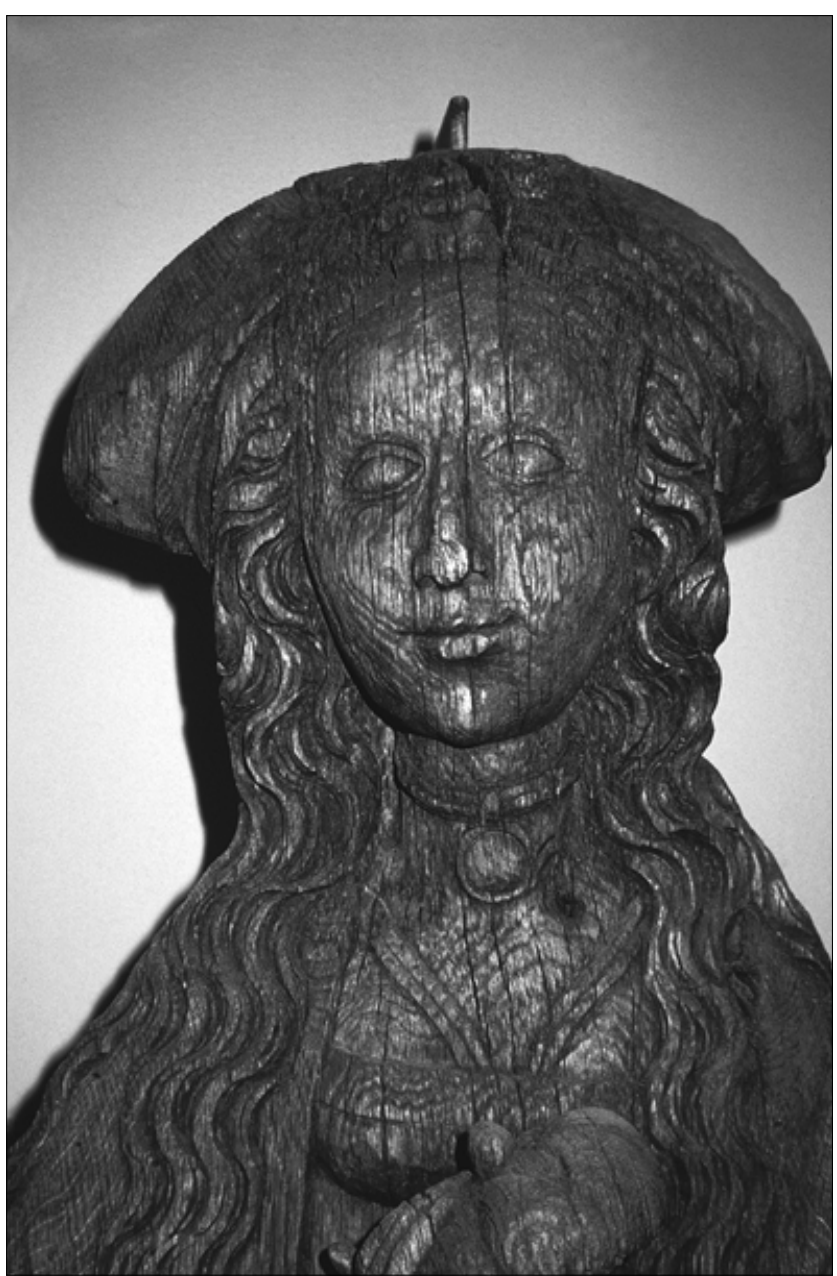

3. Escultura en madera, anónimo del Siglo XV; Oslo, Museo de Artes Aplicadas. tervenciones del ser humano. Así la documentación no puede preservar toda la información de un objeto y su máximo valor lo encierra el propio objeto en cada momento particular, y no es ni siquiera posible en teoría recuperar información ya perdida de un periodo anterior.
La reversibilidad, por tanto, es puramente una ilusión. En el mejor de los casos se adecua a restauraciones basadas en información extraída del objeto en un momento determinado. Si nuestro propósito es dar la mayor importancia a la preservación de la información de nuestro pasado, entonces está claro que cuando tratamos con objetos históricos la no intervención es la única estrategia, a pesar de que en muchas situaciones, por diversos motivos prácticos o estéticos no resulta la mejor opción. Debemos intentar preservar sólo aquello que ha sobrevivido, pero debemos ser conscientes de que intentar revertir las consecuencias del paso del tiempo no tendría éxito, excepto a nivel superficial, y estaría inevitablemente ligado a la pérdida de información histórica.

Al estudiar el principio de la reversibilidad desde un punto de vista teórico debemos, por tanto, considerar los siguientes puntos:

- La reversibilidad debe ser considerada como punto de partida de la planificación en una intervención de conservación, aunque no siempre sea posible cumplir con este principio.

- La reversibilidad no puede plantearse como principio fundamental en una intervención de conservación cuando el objeto presenta un estado de degradación extremo; en estos casos la elección del tratamiento está condicionada por la necesidad de asegurar la pervivencia del objeto en el futuro.

- El paso del tiempo es irreversible.

- Debemos trabajar en la línea de la reversibilidad, sin olvidar que el idealismo es una utopía.

- Hay que mantenerse alejados de las modas.

\section{Praxis}

Child define la restauración como "un proceso subjetivo en el que se altera la apariencia de un objeto con la finalidad de mejorar aspectos estéticos, asegurar su pervivencia o facilitar su interpretación". Sin embargo, cada vez más restauradores optan por la mínima intervención. Por ejemplo, si un objeto de metal presenta un agujero debido a un proceso avanzado de corrosión, ¿debe realmente reconstruirse antes de ser exhibido?. Hace algunos años la respuesta sería inevitablemente sí, actualmente, y cada vez más frecuentemente, podría ser no. Así, como afirma Andrew Oddy del departamento de conservación del Museo Británico, la filosofía más al día en restauración es hacer lo menos posible. Esta nueva tendencia evita en muchos casos la utilización de materiales cuya reversibilidad resulta dudosa. Sin embargo, es siempre mejor la aplicación de tratamientos no reversibles que dejar que los procesos de deterioro hagan desaparecer las obras.

La posibilidad de volver a aplicar un tratamiento de conservación sobre una pieza, "retreatibility", es más 
importante que un tratamiento aplicado anteriormente sea reversible (Appelbaum).

Ningún tratamiento de conservación directamente aplicado a un objeto de plástico deteriorado puede ser considerado reversible en su sentido tradicional.

Una vez que un plástico es calentado para ser soldado, sus propiedades físicas y químicas cambian y nunca podrán volver a su estado original. Por el contrario, técnicas no invasivas, como la introducción de absorbentes físicos en una sala de almacenaje puede considerarse reversible porque los objetos pueden volver a su estado original en las condiciones previas a su tratamiento.

Reversibilidad $=$ solubilidad $=$ hinchamiento

Jedrejewska introdujo el término pseudoreversibilidad en el sentido de "reversible en teoría pero no en la práctica". A finales de los años 60 se llegó a reconocer que muchos tratamientos no pueden ser reversibles. En la conservación de objetos arqueológicos, especialmente cuando la consolidación es necesaria, sería permisible la aplicación de tratamientos irreversibles usando materiales que hayan sido ampliamente testados" (Unesco 1968). Appelbaum en 1987 propone un planteamiento más realista afirmando que la reversibilidad "no requiere que el objeto sea idéntico a cómo era, sino que pueda volverse a un estado en el que las posibilidades de elección de un nuevo tratamiento sean tan amplias como antes de que el objeto fuera tratado"

\section{Científico}

Según este posicionamiento la reversibilidad no existe en física o en química. Siendo más importante la compatibilidad de los materiales utilizados en la intervención de restauración con los originales de la obra, que la reversibilidad de los tratamientos. Hay casos en los que la reversibilidad es una meta válida y hay otros en los que sería útil tener información sobre el grado de reversibilidad de los materiales empleados.

Así, debería intentarse cuantificar los posibles efectos a largo plazo sobre la reconocida irreversibilidad de los materiales o procesos de conservación.

\section{Lexicográfico}

Tal como mencioné anteriormente Appelbaum insiste en la confusión que existe entre los términos reversibilidad y solubilidad referido especialmente a las resinas. La solubilidad de una resina no asegura automáticamente su reversibilidad cuando es usada en un objeto. Por ello la palabra "reversibilidad" debería ser utilizada para describir un proceso más que un material.

Cuando utilizamos el término reversible referido a la intervención en obras de arte, su significado no impli-

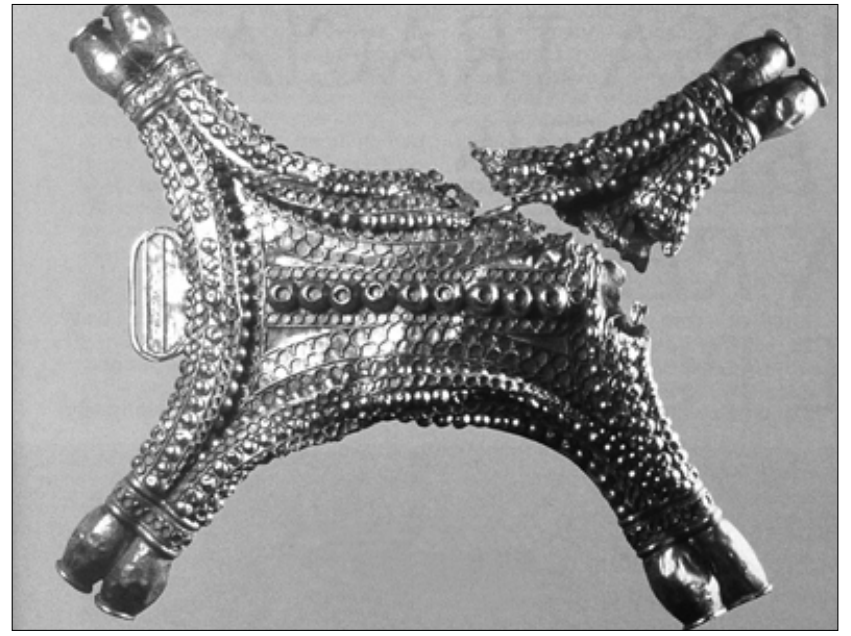

$<$

ㄴ.

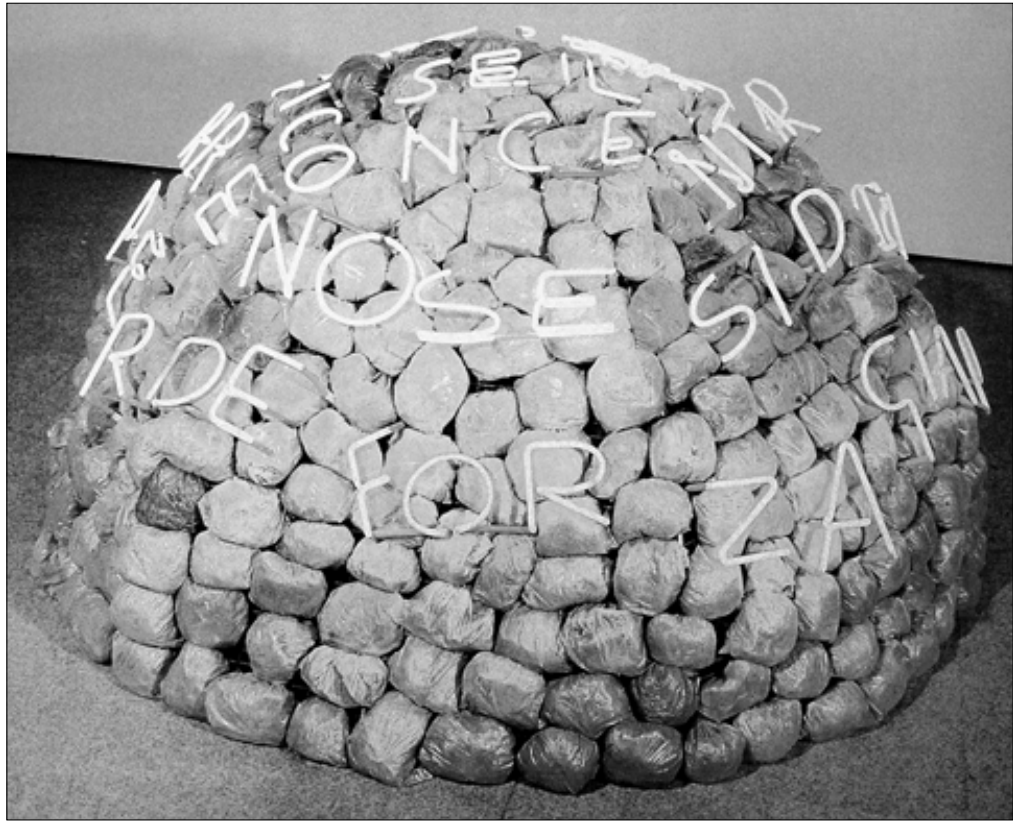

4. Anverso de pectoral con ornamentación simple. Tesoro

del Carambolo.

ca connotaciones mecánicas y, sin embargo, está más cercano al sentido de "eliminable"; por tanto se podría sugerir que la palabra reversible se sustituyera por eliminable. El uso del término eliminable presenta tres ventajas: su significado es muy claro, permite diferenciar distintos grados, y puede utilizarse cuando nos referimos tanto a procesos como a materiales.

Reversible $=$ eliminable

Es esencial para la claridad de pensamientos, así como para la integridad de nuestras acciones, que todos los términos empleados en el lenguaje de la conservación puedan ser perfectamente puestos en práctica y claramente entendidos. Así pues, Robert Barclay propone la actualización y puesta a punto del lenguaje utilizado por los conservadores, pero ciñéndonos al tema de este curso, simplemente plantea que la "reversibilidad", al menos, sea eliminada de nuestro léxico.

5. Iglú de Giap por Merz; 1968 París, Museo Nacional de Arte Moderno.

Reversibilidad = volver a tratar = "retreatability" 


\section{Conclusiones}

Para concluir me gustaría referir algunas de las ideas más aceptadas de forma generalizada por ponentes y participantes:

La reversibilidad es un sueño. En términos absolutos es imposible, pero en la práctica se lleva a cabo (Andrew Oddy).

Paradójicamente, aunque nada puede ser eliminado completamente, debemos trabajar de acuerdo al principio de reversibilidad, sin olvidar el hecho de que la reversibilidad es una utopía (Hiltrud Schinzel).

Hacer reversible un tratamiento puede ser posible en teoría, pero no en la práctica (Karen L. Pavelka).

La palabra reversibilidad podría ser irreversiblemente prohibida (Robert Barclay).

Horie ha definido la reversibilidad en cuatro niveles:

Tratamientos que no son reversibles pero que deben ser aceptados como útiles y necesarios por no encontrarse una mejor solución al problema que se plantee.
El nivel más básico es devolver el objeto al estado previo al tratamiento.

Un nivel más alto requiere que la reversibilidad del tratamiento no interfiera con ulteriores tratamientos posibles.

El nivel más exacto de reversibilidad es un tratamiento que cuando es eliminado no deja señales de contaminación del mismo y ninguna alteración de la estructura del objeto que puedan ser determinados por medios analíticos (Horie 1982).

En el desarrollo de este curso se han llegado a conclusiones y se han tomado posturas firmes y determinantes acerca de la idoneidad de la utilización del término reversibilidad, así como de la aplicación del concepto en la práctica.

La idea más apoyada es la que defiende la no existencia de la reversibilidad tanto de los procesos de conservación y los materiales empleados en ellos, como de las características intrínsecas de la materialidad de un objeto, aspecto físico o valores históricos, o dicho de otra manera la recuperación de sus propiedades tangibles e intangibles.

Bibliografía

APPELBAUM, B. 1987. Criteria for treatement: reversibility, JAIC, $26,65-73$

CHILD, R.E. 1988. Ethics in the conservation of social history objects, Prepints for the UKIC 30th Anniversary Conference, (V.Todd, ed.), UKIC, London, 8-9
HORIE, C.V. 1983. Reversibility of polymer treatements, in The Proceeding of the Symposium "Resins in Conservation" held at the University of Edimburg... 1982 (J.O. Tate, N.H. Tennant and J.H. Townsend, eds), SSCR, 3-I to 3-6, especially 3-4

A.A.V.V. 1999. British Museum Occasional Papers. Reversibility does it exist?, The British Museum, London. 